\title{
La credibilidad del enunciado científico contemporáneo: ¿última figura de la autoridad?*
}

\author{
Gépard Leclerc ${ }^{* *}$ \\ Traducción del francés al español \\ de Rodrigo Zapata Cano \\ DOl: 10.22395/csye.v6n11a17
}

De cierta manera, aunque la ciencia sea una institución milenaria, es indiscutible que el enunciado científico pertenece al centro del orden cultural moderno. Este supone la libre circulación de la información, de los datos, las investigaciones, las hipótesis, etc. y, por lo tanto, la abolición (o por lo menos la minimización de la importancia) de las censuras, la ilegitimidad de las propagandas ideológicas, que tienen por tendencia natural buscar el monopolio de la emisión de las ideas públicas. En efecto, la sociedad democrática moderna ve en los individuos sujetos enunciadores aptos y habilitados natural y culturalmente (en derecho) para expresar sus opiniones, así sean contrarias a la opinión establecida, común u oficial, las de la colectividad o las de sus representantes autorizados. Tal sociedad funciona sobre la base del reconocimiento de lo que se ha convenido en llamar, desde hace más de dos siglos, "los derechos del hombre y el ciudadano". Entre estos derechos figuran la libertad individual de la creencia religiosa, la libertad de pensar y, por ende, la libertad del examen de los discursos anteriores y actuales.

En la sociedad democrática moderna, cualquier individuo tiene el derecho de expresar su opinión. Y también podríamos decir que cualquier ciudadano tiene el deber de expresar una opinión, por lo menos sobre los problemas públicos. No obstante, aunque en el campo político todas las opiniones son legítimas, no es posible decir que todas las opiniones se valen. De manera general, existen discursos que se consideran importantes y otros que se juzgan como fútiles.

\section{Enunciados legítimos y creíbles}

Todas las sociedades, democráticas o no, reconocen algunas opiniones como las únicas legítimas, algunos enunciados como los únicos verdaderos o, por lo

Artículo tomado de la revista Esprit n. ${ }^{\circ}$ 313, marzo-abril de 2005. Traducción de Rodrigo Zapata Cano, Medellín, enero de 2017. Nota traductor.

** Sociólogo, profesor emérito en la Universidad de París VIII. Sus investigaciones se centran en los regímenes de la autoridad discusiva. Ha publicado, entre otras obras, Histoire de l'autorité, París, PUF, 1996; le Sceau de l'œuvre, París, Le Seuil, 1998; La guerre des ecritures, Fondamentalismes et laïcité à l'heure de la mondialisation, París, L'harmattan, 2009. Nota editor. 
menos, como portadores de autoridad y más creíbles que una masa de enunciados cualquiera. ¿Bajo qué criterios opera esta división entre enunciados creíbles y no creíbles, entre discursos legítimos e ilegítimos, entre discursos que se consideran verdaderos y los que se toman como falsos? Llamamos "autoridad" discursiva a una cierta forma de combinación de legitimidad discursiva y de credibilidad intelectual.

La autoridad implica primero una cierta forma de legitimidad del discurso o, mejor aún, la legitimación de algunos tipos de discursos. Las instituciones confieren la legitimidad. En la sociedad democrática moderna, el número de los discursos legítimos es enorme. Todo lo que produce la universidad, la prensa y la administración se sitúa en el campo de los discursos legítimos, puesto que de alguna manera están institucionalizados. Pero ¿cuántos de estos discursos son creíbles y considerados como portadores de la verdad? ¿Cómo se vuelven creíbles cuando se dirigen a algunos lectores o auditores? ¿Cómo se descalifican, a pesar de su legitimidad oficial, por otros lectores y auditores?

Desde hace muchos siglos en Occidente se ha producido una devaluación progresiva de los enunciados antiguos en provecho de los modernos y, asimismo, de los contemporáneos: un paso gradual, a través de crisis y mutaciones, del estatuto hegemónico de los textos de autores que pertenecen a la tradición (los antiguos, los clásicos y las sagradas escrituras) a la creación intelectual y cultural, tanto en el campo de la literatura (dominio de los "escritores") como en el de la ciencia o, como se dice en la actualidad, de la investigación. El investigador (pero también el intelectual) nace cuando los autores modernos han devaluado definitivamente la credibilidad de los autores antiguos, excepto en algunos campos específicos, que le competen a la religión, la filosofía y la literatura y según complejas modalidades que no son posibles de examinar aquí.

En las sociedades llamadas tradicionales, los discursos creíbles eran, en esencia, los que se consideraban como legítimos, en tanto garantizados por las instituciones (Iglesia y Estado) y la tradición (revelación religiosa y discursos mítico-religiosos). Con relación a este tipo de sociedad y discursos, ¿no podemos decir que la ciencia moderna representa una mutación total, que en su seno ya no existe más garantía institucional de los discursos? ¿Que el criterio de la verdad y la credibilidad son en adelante lo que se expresa en el contenido de los discursos, o más bien en algunas cualidades formales que los convierten en enunciados verdaderos? ¿En materia del establecimiento de la verdad, no es el criterio decisivo lo que Descartes llamaba "la evidencia de las razones"? ¿En la ciencia, la racionalidad como forma suprema de legitimidad de los discursos, no es inmanente a los discursos en sí mismos?

Parece que es importante discernir muchos campos enunciativos y culturales o, mejor aún, muchos campos de enunciación cultural. En efecto, en el seno de 
la sociedad moderna existen muchos tipos de "mercados discursivos", así como mecanismos institucionales que aportan modificaciones y correctivos a esta "libre circulación" de las opiniones y los discursos. De este modo, es posible distinguir un doble mercado, por lo menos en el campo cultural e intelectual moderno (Boudon):

- el mercado de los enunciados públicos (obras literarias, informaciones, ideologías, etc.) donde domina el papel de lo que se conoce como el gran público, la opinión pública y donde los controles institucionales solo se relacionan con el campo de ley y la censura política;

- el mercado (o los mercados) de los enunciados institucionales, en particular el de los enunciados científicos, donde aparecen mecanismos relativamente coactivos de homologación de los discursos innovadores y originales (sobre la base de la "libertad de pensamiento" y de la "libre competencia" entre escuelas de pensamiento, laboratorios, problemáticas, "ideologías" y paradigmas): descubrimientos, investigaciones, protocolos de experimentación, modalidades de publicación de artículos científicos, etc..

\section{La innovación científica (el "descubrimiento")}

La legitimidad de los discursos científicos es el privilegio de las instituciones. En la credibilidad, la evaluación del grado de plausibilidad de estos discursos y de la probabilidad de su verdad también se evalúa por las instituciones, pero según modalidades más complejas. Todos los discursos, para ser creíbles, deben haber pasado en general por el estado de la legitimación. De allí resulta que, por definición, todos los discursos creíbles son legítimos. Pero todos los discursos legítimos no son, ipso facto, igualmente creíbles.

Thomas Kuhn (Kuhn, 1972) es uno de los autores que ha renovado nuestra manera de considerar el funcionamiento de la ciencia como institución social y forma de control social de los discursos legítimos. En primer lugar, señala la novedad de la ciencia moderna con respecto a los discursos culturales anteriores, que descansaban sobre cierta forma de autoridad institucional; una "ortodoxia" que valorizaba los discursos establecidos y que suponían una vigilancia, cuando no una influencia del poder político, sobre los discursos públicos. Existe una autonomía de la ciencia moderna y de la comunidad científica con relación a todas las autoridades exteriores e institucionales: Estados, iglesias, etc., y también en conexión con el poder anónimo de la colectividad que se expresa como consenso establecido, de las tradiciones establecidas o de la opinión pública. Parece que en la democracia ninguna autoridad institucional llega a interferir en los debates libres en la comunidad científica. La ciencia misma es 
una autoridad institucional: tiene sus autoridades, instituciones, representantes oficiales, referencias, creencias, su tradición y ortodoxia...

Aunque Kuhn reconoce que la investigación de la innovación, es decir, de los "descubrimientos", es el motor de la actividad científica, pone en entredicho la idea estereotipada según la cual la ciencia moderna sería fundamentalmente investigación, descubrimiento e innovación. Es evidente que los "científicos" se dedican a hacer descubrimientos y que la ciencia es una forma de "investigación". También es cierto que la innovación se valoriza a expensas de los discursos establecidos y antiguos. Sin embargo, Kuhn parece insistir sobre todo en los factores de inmovilismo y conservadurismo que la caracterizan. La ciencia no es fundamentalmente innovación, investigación y descubrimiento, es memoria, tradición y certeza. Como sabemos, el concepto de paradigma asociado al de "ciencia normal" expresa esta propiedad central del discurso científico.

El problema fundamental que Kuhn plantea es el siguiente: ¿Cómo se pasa de un sistema de creencias bien establecido e institucionalizado (tradición) a una remodelación total del sistema de creencias (revolución científica)? Kuhn responde: por medio de una mutación más o menos rápida, brutal y progresiva de las creencias propias de la comunidad científica en su totalidad y, por lo tanto, de cada uno de sus miembros. Un descubrimiento consiste en evidenciar un hecho nuevo; una invención es la producción de una nueva teoría; la "revolución científica" es un cambio de paradigma.

Un paradigma es un modo reconocido de trabajo científico que provee modelos aptos para dar nacimiento a una tradición particular y coherente de la investigación científica. De esta manera, en la Edad Media pudo reinar por completo la astronomía de Ptolomeo, antes que la de Copérnico la destronara. La física aristotélica pudo ejercer su hegemonía desde la Antigüedad al Renacimiento y también en la Época Clásica.

Por lo demás, el término paradigma designa a la vez la comunidad científica (la colectividad de los investigadores en conjunto y que existen en un momento dado) y la institución científica, cuya función es transmitir la tradición científica, así como la tradición científica en sí misma. Estos tres sentidos están ligados porque el paradigma como "régimen de verdad" es el resultado de un conjunto de procesos que se desenvuelven únicamente en la comunidad científica: "La elección del paradigma no se puede imponer por ninguna autoridad superior al consentimiento del grupo" (Kuhn, 1972: 136). El paradigma que predomina en un momento dado es el resultado de un consenso que se establece después de libres debates y discusiones y, por lo tanto, de disensos en la comunidad científica.

Los científicos trabajan siguiendo reglas, fundándose en modelos que proceden de sus estudios o de informes de trabajos recientes. Así pues, experimentan 
un aprendizaje que es un "modelado" sobre las "autoridades" y la tradición. Por consiguiente, aquí desempeña un papel fundamental lo que Kuhn denomina "la autoridad de los profesores y los manuales" (Kuhn, 1972, p. 118). En efecto, el trabajo científico "normal" se apoya en la utilización de "fuentes autorizadas": los manuales científicos, las obras de vulgarización, así como los trabajos filosóficos que se basan en ellos. Estas fuentes, vectores centrales de la historia oficial de las ciencias, minimizan las discontinuidades que en realidad han existido a lo largo de la historia de las ciencias y maximizan la apariencia de un progreso continuo y lento, pero regular, de la ciencia. La tradición, tal como se difunde a través de los manuales, es una versión empobrecida, edulcorada y algunas veces francamente inexacta, de la historia de las ciencias. Los manuales nos dan una formulación estandarizada de los descubrimientos pasados. Ahora bien, el investigador medio toma consciencia de la historia de su disciplina solo a través de estos manuales. Es la total diferencia entre el estatuto de las ciencias naturales y el de las ciencias del hombre o de las disciplinas artísticas (humanities), que practican una recolección permanente de su historia y memorizan y tamizan sin descanso sus tradiciones y fuentes: "No existe nada en la formación científica que sea el equivalente del museo artístico o de la biblioteca de los clásicos" (Kuhn, 1972, p. 228).

La consecuencia es una profunda distorsión entre la historia real de las ciencias y su historia oficial o institucional.

\section{La homologación de los descubrimientos}

El problema de la credibilidad se plantea muy poco en el campo de los saberes establecidos y en los paradigmas dominantes compartidos por la comunidad científica o, por lo menos, por la gran mayoría de sus miembros. El saber científico es impersonal, anónimo, y se considera fiable. Por el contrario, el problema de la credibilidad se plantea en la franja de la enunciación científica y en el campo de la innovación discursiva, en particular cuando pone en entredicho la existencia y la naturaleza de los paradigmas existentes.

Tal parece que en Kuhn existen dos sentidos algo diferentes del término paradigma. En efecto, este designa: de una parte, "el conjunto de creencias, valores reconocidos y técnicas que son comunes a los miembros de un grupo dado". Se trata de una definición "sociológica" del paradigma, nos dice Kuhn. Entiende por este término la red de comunicaciones: conferencias, artículos, citas, bibliografía común, autores de referencia, escuela de pensamiento, etc. Este sentido es muy parecido a lo que Foucault entendía como "régimen de discurso"; de la otra, las nuevas soluciones que se aportan para resolver enigmas en el marco de la tradición científica. En síntesis, el término designa más bien un nuevo "régimen de verdad". 
Se pueden distinguir dos tipos de discursos en el mundo científico:

- los discursos establecidos que, por definición, gozan de buen crédito y de una autoridad indiscutible;

- los discursos innovadores (supuestos descubrimientos, invenciones teóricas, etc.) que constituyen el "frente de la investigación", es decir, la línea de combate de los investigadores contra los "secretos" de la naturaleza.

Aquí la enunciación científica es original y nueva, la mayoría de las veces o, por lo menos, es lo que se quiere: se presenta como una "apertura", un importante descubrimiento, esencial y fundamental. ¿Cómo opera la legitimación del descubrimiento?

La innovación primero se reivindica públicamente por el investigador en el campo de la investigación y en la especialidad que le compete, en tanto que miembro competente de la comunidad científica y cuyo grado de credibilidad es, por principio, igual al de todos sus colegas y competidores. Su reivindicación de conocimiento o su pretensión de saber (Knowledge claim, como dicen los sociólogos y filósofos anglosajones) está legitimada en derecho por la naturaleza misma del discurso, por el contenido del supuesto "descubrimiento", independientemente de su estatuto profesional e institucional en la comunidad científica: poco importa si es un investigador conocido o desconocido, un miembro medio y anónimo de un equipo de investigación poco conocido o, por el contrario, el director de un importante laboratorio, conocido por sus trabajos anteriores y también por descubrimientos importantes. En virtud del credo democrático (todos los hombres son libres e iguales en derechos), todos los enunciadores científicos (todos los miembros por derecho de la institución de la investigación) tienen la posibilidad de reivindicar una fracción de la autoridad enunciativa. Existe una igualdad, por lo menos formal, entre los miembros de la comunidad científica, en el plano de los derechos de enunciar un discurso innovador y en el de los derechos de criticar los pretendidos descubrimientos de otro y su reivindicación de saber (knowledge claim).

Pero este solo es el primer paso en el proceso de validación y legitimación del "descubrimiento", que conducirá a su eventual aceptación y apropiación por la comunidad científica. Pues el descubrimiento debe ser objeto ahora de una evaluación y legitimación colectiva e institucional. Del otro lado de las reacciones individuales, el ejercicio de la facultad crítica les pertenece por derecho a la comunidad de los investigadores y a la ciencia como institución nacional, pero también y sobre todo, internacional, transcultural, no religiosa y transconfesional. ¿De acuerdo a qué criterios opera esta evaluación de la "verdad" o de la falsedad del enunciado nuevo? ¿Es preciso también hablar de la verdad del 
enunciado? En su obra sobre las revoluciones científicas, Kuhn presume de no haber empleado ni una sola vez la palabra "verdad". Dicho de otro modo, lo que está en juego no es tanto la verdad pura y simple, como la probabilidad de la verdad del enunciado, su plausibilidad y credibilidad.

\section{La credibilidad de los descubrimientos}

Se deben tomar en cuenta dos puntos aparentemente contradictorios. A pesar de su pretensión en el rigor lógico del contenido y de las reglas formales de su producción, la mayor parte de los enunciados innovadores, aunque buscan obtener la aprobación de los pares científicos, no mantienen sino una débil probabilidad de verdad.

Desde luego, no todos los enunciados se consideran equiprobables. Pero no existe medio infalible, seguro y cierto, de determinar de una vez por todas la validez y la "verdad" de un enunciado nuevo. La única operación posible y razonable, que se practica efectivamente por los investigadores, es el principio que Popper llamó "falsificación" en su obra la Lógica del descubrimiento científico (Popper, 1973), (se podría preferir la traducción "refutación" por el término homónimo inglés). No se puede demostrar la verdad de un enunciado. Lo mejor que se puede hacer es demostrar su falsedad (es lo que él denomina falsificación). El criterio de la "cientificidad" de un enunciado, es decir, de su pertenencia al universo de la "ciencia" no es otro que su "falsifiabilidad". Un enunciado científico es un enunciado empírico o experimental que se somete a un test de verificación, una prueba de falsificación, un experimento crucial o una demostración lógico-matemática susceptible de ser "falsificada".

Esta tesis fue elaborada a partir de una observación por medio de la cual Popper estimaba el trabajo efectivo de los investigadores modernos en el campo de las ciencias naturales, donde existen reglas y procedimientos de verificación / falsificación sofisticadas, establecidas desde hace decenios y compartidas por toda la comunidad científica internacional. ¿Pero qué ocurre con las disciplinas que llamamos, tal vez de manera exorbitante, las "ciencias del hombre"? Aquí, la "comunidad" de los investigadores está dividida por las escuelas, las problemáticas, las ideologías rivales y conflictivas. En este campo, parecen existir universos de discursos y regímenes de verdad difícilmente compatibles y asimilables a un paradigma único y a un modelo de pensamiento establecido y dominante.

En las "ciencias del hombre", parece predominar no tanto lo probable y lo verosímil, sino lo plausible y lo que parece verdad (vraisemblant). La verdad parece residir solo en un efecto retórico, que podríamos llamar "el efecto de verdad". La cualidad científica del enunciado parece reducirse a su valor simbólico, estimado 
en términos sociales y donde la institución científica parece perder sus puntos de referencia tradicionales, los que funcionan precisamente en las ciencias naturales. Aquí, la mayor parte de los enunciados son no-falsificables (Passeron, 1990). En el mejor de los casos, sin ser manifiestamente falsos, presentan una cierta apariencia de credibilidad. En el peor de los casos, son simplemente "interesantes", nuevos, plausibles y posiblemente ciertos. No obstante, sin que exista la posibilidad de atribuirles una verdad o falsedad indiscutibles, incluso la calidad de discursos refutables o verificables, también desde el estricto punto de vista de la "falsifiabilidad" popperiana. La plausibilidad supone cierta credibilidad, pero limitada e inverificable.

En este club tan cerrado que es el "frente de la investigación" existe un pequeño número de enunciadores competentes y susceptibles, por su experiencia profesional y por la calidad de sus anteriores trabajos, de producir enunciados verdaderos o, por lo menos, plausibles. Pero aquí también es restringido el número de quienes están en condiciones de evaluar la credibilidad del enunciado innovador, del "descubrimiento", de aprobarlo y apropiárselo. Solo el tiempo permitirá decir, casi de seguro, lo que realmente es y hará que, poco a poco, nuevos lectores especialistas lleguen a reconocer la "verdad" del descubrimiento. Son raros los críticos competentes y los lectores calificados para formular rápidamente, y en el campo, un juicio evaluativo motivado por la calidad del enunciado innovador que emita su knowledge claim. En el límite, en algunos campos que se denominan "puntiagudos", un solo especialista, lector-enunciador único, estará en condiciones de dejarse persuadir por las razones del enunciado innovador. El hermetismo y la complejidad del discurso ocultan la "evidencia de las razones" que, en principio, desde Descartes, gobiernan el régimen de la enunciación científica. ¿Son geniales un artículo de biofísica o una obra de cosmología? O ¿son una exageración? O ¿una estafa intelectual?

Después de la evaluación "en caliente" del valor diferencial de los nuevos enunciados (de los "descubrimientos"), debe intervenir otro juego de variables como la credibilidad interna, propia del enunciado. Este juego de variables suplementarias, de tipo sociológico, es el que tiene que ver con la credibilidad diferencial de los investigadores. ¿Cómo pueden los enunciadores, iguales en derechos, diferenciarse en cuanto a la calidad de su contribución, la fiabilidad de sus enunciados y al valor de sus pretensiones científicas?

\section{La credibilidad de los investigadores}

La sociedad tradicional estaba dominada por el principio de la auctoritas, la cual se fundaba en el valor durable de las ideas antiguas. En la modernidad científica, la "credibilidad" del investigador, del experto y del especialista se vuelve el criterio y el problema esencial, cuando se trata de la validación y la 
homologación de los discursos y, en particular, de los discursos innovadores. Para comprender el estatuto y la significación de la noción de credibilidad, aplicada a los individuos y no a los discursos, es importante analizar el concepto de creencia y, por lo tanto, el significado del verbo "creer".

Ningún discurso se sostiene por sí mismo; solo se vuelve creíble por la única evidencia de sus razones, excepto, tal vez, en algunos campos de las ciencias matemáticas. Todos los discursos, sobre todo cuando son innovadores, se deben remitir a una fuente, atribuirse a un "autor", a su autor en el sentido de agente, creador y fuente, a quien los ha proferido y que profiere, al mismo tiempo, un knowledge claim. Esta fuente y autor son los que constituyen, por lo menos durante un primer momento, la última garantía de la veracidad del discurso.

En efecto, el verbo "creer" algunas veces se relaciona con las cosas, con el objeto de la creencia. En este sentido decimos: "creer que..." (en un hecho, una cosa, una realidad, una verdad o un discurso). El verbo "creer" también tiene que ver con personas. Entonces decimos: "creo en tal" (en sus actos, palabras, etc.). Existe cierta irreductibilidad de estas dos formas de "creencia". La primera se enlaza con la opinión como doxa, mientras que la segunda se vincula con la confianza como fe (pistis). Ahora bien, se ha podido sostener con alguna verosimilitud que el "creer que" supone siempre (algunas veces implícitamente) un "creer en".

Asimismo, el discurso científico contemporáneo que, por lo menos en apariencia, constituye un "saber", muy a menudo forma un "creer". Al tratarse de enunciados innovadores y supuestos descubrimientos, este último es a la vez un "creer que" y un "creer en". Por lo tanto, es verdadero, y se pondrán en marcha procedimientos de verificación / falsificación, que conducirán ya sea a un rechazo del discurso como pseudo-descubrimiento (véase la famosa controversia sobre la "memoria del agua"), o a su homologación como discurso científico verdadero. A partir de este momento, podrá ser anonimizado, es decir, separado de su autor. En lo sucesivo, la credibilidad del discurso será independiente de la de su autor, la cual se volverá transitoria y obsoleta ${ }^{1}$.

No obstante, tratándose de los regímenes generales de enunciación científica, de estas grandes teorías arquitectónicas que constituyen los paradigmas científicos, la credibilidad de sus primeros autores parece persistir oculta como última garantía y también en el plano histórico y puramente simbólico. Se habla de la astronomía de Ptolomeo y Copérnico, de la física de Newton y Einstein, etc.; como si la autoridad de estos garantes continuara siendo el último soporte de

Además de la obra de K. Popper, véanse los trabajos de Bruno Latour y Stève Woolgar, en particular la Vie de laboratoire. La production des faits scientifiques, París, La Découverte, 1988. [La vida en el laboratorio. La construcción de los hechos científicos, Madrid, Alianza 1995]. 
su legitimidad y credibilidad. Por lo demás, es un nuevo autor, un investigador innovador, quien pondrá en entredicho la credibilidad del paradigma, reivindicará una credibilidad propia y nueva, y se opondrá al paradigma existente, al autor-autoridad reconocido hasta entonces como verídico. Kuhn admite, en La estructura de las revoluciones científicas, el papel primordial de la autoridad, en particular la importancia del nombre del investigador, de su notoriedad y credibilidad institucional en la publicación, difusión y aceptación de su "descubrimiento" (Kuhn, 1972: 210). Insiste en el papel de la confianza y la fe (Kuhn, 1972: 216). En las ciencias sociales en particular, la innovación se acepta después de una modificación de la "distribución de las persuasiones profesionales", donde la credibilidad y la notoriedad de los investigadores desempeñan un papel primordial. Esta importancia es menor en las ciencias de la naturaleza. Kuhn llegará a decir también que, en las ciencias sociales interviene como criterio determinante la notoriedad del investigador con respecto al gran público; entre los profanos, la notoriedad en la cual los medios de comunicación desempeñan un importante papel: pensemos en la audiencia de tal o cual intelectual conocido. Mientras que en las ciencias naturales el criterio de la credibilidad científica es la notoriedad en el seno de la comunidad científica y la credibilidad frente a los colegas y pares.

En resumen, la reivindicación de la autoridad (otro nombre para la "reivindicación de saber", para el Knowledge claim) supone muchas etapas o variables para lograr el éxito:

- la legitimidad de la reivindicación;

- la investigación (después la obtención) de garantías, que son de tipo intradiscursivo (racionalidad y evidencia propia del enunciado, naturaleza y calidad de la intertextualidad como red elaborada de citas, referencias, etc.), pero también de tipo extradiscursivo (naturaleza y notoriedad de la revista donde fue publicado el artículo, notoriedad de la universidad o del instituto donde trabaja el investigador; más generalmente, el cargo profesional y reconocimiento institucional del investigador).

\section{La importancia de la firma: marca del autor y de la verdad}

Solo en el campo de los discursos científicos establecidos y admitidos por la comunidad científica como verdades indiscutibles (paradigmas), los enunciados parecen sostenerse por sí mismos y no necesitan ninguna garantía individual o colectiva. En este sentido, el enunciado paradigmático es anónimo: ha perdido sus marcas de origen; en particular, el nombre de su autor y las indicaciones sobre su fuente. Las conserva raras veces, por medio de una 
rememoración o memorización "artificial" y voluntaria, que Merton propuso llamar "eponimato": se trata de la conmemoración oficial y solemne del nombre del inventor, descubridor y teórico genial, a través de la certificación de una innovación intelectual y un nombre propio. Todos los enunciados innovadores permanecen asociados al nombre de su inventor o descubridor: la nueva ley científica, el nuevo teorema, el nuevo principio ("curva de Gauss", "teorema de Fermat", "principio de Gödel"), el descubrimiento de una nueva técnica (la "pasteurización") o el descubrimiento o análisis de una "nueva" enfermedad ("enfermedad de Alzheimer"); todos estos enunciados innovadores perduran asociados al nombre de su inventor o descubridor. Pero, por fuera de estas excepciones, cuyo carácter honorífico y original salta a la vista, la enunciación científica estandarizada, que se sitúa en el interior de un paradigma, permanece totalmente anónima, disociada de cualquier marca individual distintiva y mención sobre su fuente. De este modo, la astronomía copernicana se vuelve la astronomía a secas; la física newtoniana se convierte en la física moderna; la genética mendeliana se vuelve pura y simplemente la genética. El descubrimiento, que en otro tiempo era un discurso problemático, se convierte en "paradigma".

No sucede lo mismo en el caso de los enunciados innovadores y los "descubrimientos" eventuales, que en realidad se presentan primero como Knowledge claims, reivindicaciones de nueva verdad. Además, en un primer momento, la identificación del autor, de la fuente del discurso problemático, aparece como una necesidad, tanto lógica como sociológica. Es necesario que el lector, el colega y especialista del campo correspondiente, conozcan el nombre y sobre todo la identidad institucional del supuesto descubridor: nombre de la universidad, del instituto de investigación, etc. Así, en el frente de la investigación, el nombre vuelve a ser importante. Permite al lector (colega, especialista y crítico) intentar evaluar una reivindicación de autoridad y su pretensión de haber descubierto algo nuevo en el campo de la verdad científica, por medio del nombre y la posición institucional del autor, los títulos de acreditación, la credibilidad y la legitimidad de su derecho para emitir un knowledge claim: un nuevo principio, una nueva fórmula, una nueva ley, un nuevo teorema, un nuevo hecho, etc.

Así pues, aparece un importante problema sociológico. Los mecanismos de poder institucional (administrativo y simbólico) ¿no se arriesgan a interferir en una evaluación "neutra" y "objetiva" de la calidad de las diferentes contribuciones individuales y el valor relativo y respectivo de los discursos y sus autores, de los investigadores y su "producción"?

Tomemos el caso de un enunciado nuevo, cuyo autor es "desconocido", es decir, cuyo nombre y estatuto no sostienen ninguna marca positiva de notoriedad 
ya establecida: sin duda, un autor joven que todavía no ha hecho ningún descubrimiento o no ha producido ningún trabajo importante. De esto se desprende que, como primera consecuencia sociológica, la "visibilidad" de su discurso será débil. El artículo que presenta el "descubrimiento" se arriesga a ser ignorado por completo, no ser objeto de ninguna evaluación crítica (review) en las grandes revistas científicas y no ser discutido por los especialistas, etc. No obstante, es posible que el artículo en cuestión llegue a leerse y apreciarse, a pesar de esta desventaja del comienzo, teniendo en cuenta sus cualidades intrínsecas de novedad, sobre todo la fuerte credibilidad propia y defendiendo la calidad superior del contenido. También puede ocurrir que sea ulteriormente reconocido por anunciar un gran descubrimiento. De esta manera, el enunciado será evaluado positivamente, considerado como creíble o, para hablar de modo más riguroso, acreditado en un porcentaje elevado de credibilidad y autoridad, luego del examen de las propiedades internas, de las características lógicas propias y después que el tiempo haya desempeñado su papel de tamiz de las cualidades diferenciales que lo distinguen del "recién llegado" de la producción científica.

Es posible entonces que el nombre de su autor llegue a adquirir también una credibilidad diferencial elevada y una autoridad. Y esta nueva credibilidad y autoridad alcanzadas llegarán a desempeñar un papel inicial mayor, cuando se trata de juzgar, evaluar, criticar la futura producción del investigador, la calidad de los nuevos enunciados producidos por él y la credibilidad de sus nuevos Knowledge claims. Un enunciado cuyo autor tiene un nombre conocido desempeña un juicio favorable, es más "visible" en la comunidad científica: ha sido publicado en las revistas más prestigiosas, es más notable que los artículos de autores desconocidos. En consecuencia, se juzga a priori como más creíble que el promedio de los nuevos enunciados científicos. Un enunciado cuyo autor es desconocido (con un nombre "desconocido" y "que no les dice nada a los especialistas) no es "neutro" en términos de crédito: tiene un "crédito negativo" a priori, está afectado por un índice de credibilidad débil o nula. No hay discursos cuyo valor simbólico sea "neutro" y, en particular, los discursos científicos. No existen nombres cuyo valor simbólico no se contabilice de manera positiva o negativa. No existen nombres situados fuera de la evaluación simbólica. Así pues, se pueden oponer dos tipos de nombres mayores:

- los "grandes nombres", los de personalidades científicas, autoridades, investigadores conocidos, "solemnidades intelectuales", etc.: los discursos que acompañan y a los que están asociados por authorship son a priori valorizados y "credibilizados";

- y los nombres "sin valor" (o con un valor negativo), los que, no sin algo de menosprecio, algunas veces se denominan los "anónimos": los discursos que firman son a priori descalificados, desvalorizados o subvalorados. 
Merton, uno de los grandes sociólogos especialistas de la producción científica, estudiando las condiciones sociales de la creación y difusión del enunciado innovador (Merton, 1973), evidenció un fenómeno que denominó el "principio de Mateo". Este principio enuncia que los investigadores conocidos ven sus nuevos enunciados acreditados a priori en un porcentaje elevado de credibilidad, mientras que los trabajos de los investigadores desconocidos tienen probabilidades de ver sus trabajos ignorados o considerados sin valor. El nombre de este principio fue tomado de una frase del evangelio de Mateo: "al que tiene se le dará y abundará; pero a quien no tiene se le quitará".

Resumamos: el nombre de autor (en este caso el nombre del investigador, del enunciador científico que pretende producir una innovación intelectual) afecta el discurso en un índice dado de verdad o plausibilidad. Designa un porcentaje de credibilidad dado. El nombre del investigador conocido goza de notoriedad, es un sello de calidad.

En “¿Qué es un autor?" y en La arqueología del saber, Michel Foucault (Foucault, 1969, 73-104; 1969) reconocía que no era fácil librarse de los nombres de autores, aún en el marco del trabajo arqueológico que se interesaba por la estructura anónima de los enunciados, de las epistemes, y no en autores y obras. El mérito de Foucault ha sido transformar evidencias (el "autor" y la "obra") en objetos de investigación filosófica, histórica y sociológica. Pero sus profecías (semejantes a las de Barthes) sobre "la muerte del autor" se han revelado muy problemáticas. En efecto,

- ¿Cómo explicar a la vez la problematización reciente del nombre de autor, del lugar entre los enunciados, la supuesta fuente y la asignación (atribución) de los discursos a un "autor"?

- ¿Cómo explicar la universalidad de la firma, de la atribución cultural de los discursos colectivos a una supuesta fuente, individualizada o colectiva, humana o divina? ¿Cómo explicar los mecanismos por los cuales una cultura asigna sus textos y clásicos, ya sea a una fuente humana individualizada, que adquiere un estatuto casi sobrehumano (Buda, Confucio, Platón, etc.), o a una fuente de entrada considerada como suprahumana, divina, sagrada y trascendental (Dios), mediada históricamente por una individualidad humana como la del Profeta?

- ¿Cómo explicar la persistencia, en la investigación científica contemporánea, de la importancia de los nombres de los autores (investigadores, descubridores) y de la notoriedad en tanto ligada a la credibilidad y la autoridad? 
$\mathrm{El}$ objetivo de algunas de las observaciones anteriores era simplemente poner en entredicho y problematizar el hecho sorprendente -por lo menos en apariencia- de que los enunciados científicos establecidos (paradigmas) tienen la propiedad de poder ser anónimos, de estar en condiciones de "pasarse de firma". También intentamos establecer como digno de análisis histórico y sociológico el problema de la universalidad de la firma, de su papel en la construcción de la autoridad discursiva, como garantía de la verdad o, por lo menos, de la credibilidad de los enunciados nuevos. En efecto, el problema no es despreciable. En primer lugar, se trata de evidenciar la generalidad de una evaluación de los enunciados y una jerarquía de los discursos. Y en segundo lugar, los principios (o al menos algunos de ellos) que dirigen esta evaluación y jerarquización. Desde este punto de vista, se podrían distinguir dos casos extremos: de una parte, el Libro sagrado y, de la otra, el discurso ordinario. Entre ambos, una pluralidad de gradaciones y un gran número de casos intermediarios, que varían según las culturas y las épocas históricas y los "géneros discursivos" institucionalizados en los diferentes tipos de sociedades: clásicos, teología, literatura, ciencias, saberes, periodismo, opinión pública, discursos cotidianos... El problema de una sociología de la autoridad de los discursos se sitúa entre dos extremos que son la sociología de la religión (o de la tradición) y la sociología de la ciencia (y de los medios de comunicación).

\section{Bibliografía}

Boudon, R. "Les deux marchés", en Encyclopædia Universalis, suplemento, sin fecha.

Foucault, M. (1969). "Qu'est ce qu'un auteur", sesión del sabado 22 de febrero de 1969, en Bulletin de la société française de philosophie, t. 64. [“¿Qué es un autor?”, Obras esenciales, Vol. I, Barcelona, Paidós, 1999].

(1969). l’Archéologie du savoir, París, Gallimard. [La arqueología del saber, Buenos Aires, siglo XXI, 2008].

Kuhn, T. (1972). La Structure des révolutions scientifiques, París, Flammarion. [La estructura de las revoluciones científicas, México, F.C.E, 1998].

Latour, B. y Woolgar S. (1988). La Vie de laboratoire. La production des faits scientifiques, París, La Découverte. [La vida en el laboratorio. La construcción de los hechos científicos, Madrid, Alianza, 1995].

Merton, R. (1973). The Sociology of Science, Theoretical and Empirical Investigations, Chicago, Londres, The University of Chicago Press. [Sociología de la ciencia, Madrid, Alianza Editorial, 1977].

Passeron, J. (1990). Le Raisonnement sociologique: l'espace non poppérien du raisonnement naturel, París, Nathan.

Popper, K. (1973). La Logique de la découvert scientifique, París Payot. [La Lógica del Descubrimiento Científico, Madrid, Tecnos, 1965]. 